\section{Location of errors with a post-stimulus indicator ${ }^{\text {? }}$}

RALPH NORMAN HABER and LIONEL STANDING, ${ }^{2}$ University of Rochester, Rochester, N.Y. 14627

To examine why Averbach \& Coriell (1961), in their experiment on short-term visual storage, found a " $W$ "-shaped error distribution over positions, their experiment was replicated, using as stimul arrays of one row of eight letters, each followed by an indicator delayed from 0 to 3,200 msec informing $S$ which of the eight letters to report. Further, on half of the 2,560 trials, parentheses were drawn around the array to simulate an equivalent amount of metacontrast for the end letters as for all of the others. The " $W$ " shape was replicated with and without parentheses, although adding parentheses substantially reduced the accuracy of Positions 1 and 8 , leaving the others virtually unaffected. No interaction was found between delay and position, nor did any change in the properties of visual or auditory confusion among the errors occur over positions, delays, or parentheses. The results all suggest that the " $W$ " function is due strictly to better acuity for the middle items near fixation and less metacontrast for the end items. No hint was found that the pattern of errors might be due to processes related to encoding or to memory-maintenance variables.

Averbach \& Coriell (1961) presented 16 letters arranged in two rows of 8 for $50 \mathrm{msec}$ at $70 \mathrm{ft}-\mathrm{L}$ under light-adapted conditions. Following a variable delay, a bar indicator appeared below or above 1 of the 16 positions and $S$ was instructed to report or to guess the letter that had occupied that position. They found that the probability of correctly reporting any letter indicated was a decaying function of the interstimulus interval (ISI) between the array and the indicator, reaching a minimum accuracy at about $1 / 4 \mathrm{sec}$. In an error analysis, summed over all ISls, greatest accuracy was found for the positions in the middle of the array (those nearest the fixation point) and for those at each end. This was true for both the top and the bottom row, though for all positions Ss were most accurate on the top row.

Since this "W" function is unlikely to be due to a single process, the following simple (though by no means brief) experiment was undertaken to examine some of its potential components. These include: better acuity for the items nearest fixation; less metacontrast for the end items, since they are the only items not surrounded on both sides by other items (this is tested by placing parentheses around the array); earlier scanning of the end items, so that they are processed while their visual storage is more adequate; or better resistance of the end items to serial-position interference effects of the kinds that underlie most bow-shaped serial-position functions.

\section{SUBJECTS}

Two University of Rochester students served as paid Ss. Both were initially unpracticed in psychophysical tasks. Each served in 25 ith sessions, the first 5 of which were for practice. Three other Ss began the experiment but dropped out in its early stages. Their data are not included in these analyses.

\section{STIMULI AND APPARATUS}

Figure 1 shows an example of an eight-letter array with and without parentheses. An indicator is also drawn, though in actuality this was superimposed from another channel. The arrays were drawn with a Leroy lettering set, No. 3240-350CL, with a No. 4 pen. All letters were used except $Q$. Each array was printed on a white card ( $90 \%$ reflectance), which was displayed at the rear of Channel 1 of a three-channel Scientific Prototype Model GA tachistoscope. Each indicator was printed on a card displayed in Channel 2. The third background channel contained a similar card, with two faint horizontal lines above and below the array to indicate a fixation region. All channels were set at $20 \mathrm{ft}-\mathrm{L}$. The background channel was on at all times except when $S$ initiated the trial, when it was replaced by Channel 1 for $50 \mathrm{msec}$. The background channel returned for the appropriate ISI, whereupon Channel 2 containing the indicator came on for $50 \mathrm{msec}$. The background channel then returned until the next trial commenced. All timing and sequences were controlled by a PDP-8 computer, on line with the tachistoscope (see Haber, 1968). Eight ISIs were used: simultaneous, 0 (immediately successive), 50,100,300,800,1,600, and $3,200 \mathrm{msec}$. For the simultaneous ISI, the

$$
\begin{aligned}
& -4^{4.2}-1 \\
& \text { ( K Y J Z K T O C ) \$0.370 } \\
& \left.\left.\rightarrow\right|_{0.26^{\circ}}\right|^{\left[0.37^{\circ}\right.}
\end{aligned}
$$

Fig. 1. An example of a stimulus array with parentheses. The dimensions are in visual angles. indicator was drawn on the array card, and Channel 2 was not used.

A random generator program created 2,560 arrays of eight letters, sampled with replacement from 25 letters of the alphabet. For each array, two numbers from 1 to 8 were also assigned to specify the position to be indicated and the ISI. The 2,560 arrays, with their associated position and ISI, were contained in the PDP-8's memory. For each session (of about $1 \mathrm{~h}$ ), 128 arrays were presented, representing two instances of each position-ISI combination. For each $S$, the practice sessions and the first five sessions were without parentheses drawn on the arrays; the next 10 sessions were with parentheses, and the last five sessions were again without. Each $S$ received the 20 sessions in the same order. Except for the parenthesis manipulation, each session was a complete replication of the experiment.

Before each session, E would place the two sets of 128 cards to be shown (arrays and indicators, respectively) in the appropriate channels of the tachistoscope and would indicate to the PDP-8, via a Teletype next to the tachistoscope, the session number (from 1 to 20) being run. The printer would then type the session number and the trial number (from 1 to 128). When the printing ended, $S$ knew he could initiate the trial. After the trial was over, the printer typed out the eight letters that had been shown in the array, the position and letter indicated, and the ISI. When the $S$ made a verbal report (giving the letter and position indicated), E would enter these, and they would also be typed out, continuing on the same line. The printer then automatically moved to the next line, setting up the next trial. At the end of the 128 trials, two summary tables of per cent correct for each of the 64 cells were computed: one for that block of 128 trials alone and then a cumulative table for that block plus all previous blocks of the appropriate parenthesis condition (the data having been maintained in computer memory).

\section{RESULTS}

Figure 2 shows the effects of parentheses and position on accuracy and Fig. 3, the effects of parentheses and ISI on accuracy, each for the two Ss separately. Since an analysis of variance indicated that no-position by ISI effect (two- or three-way) reached significance, these figures can be considered independently. In Fig. 2, the Position by Parentheses effect was highly significant $(F=11.9$, df $=7 / 49, p<.01)$, and resulted primarily from lower performance in Positions 1 and 8 when the parentheses were added. Thus, the absence of a metacontrast effect in the original 


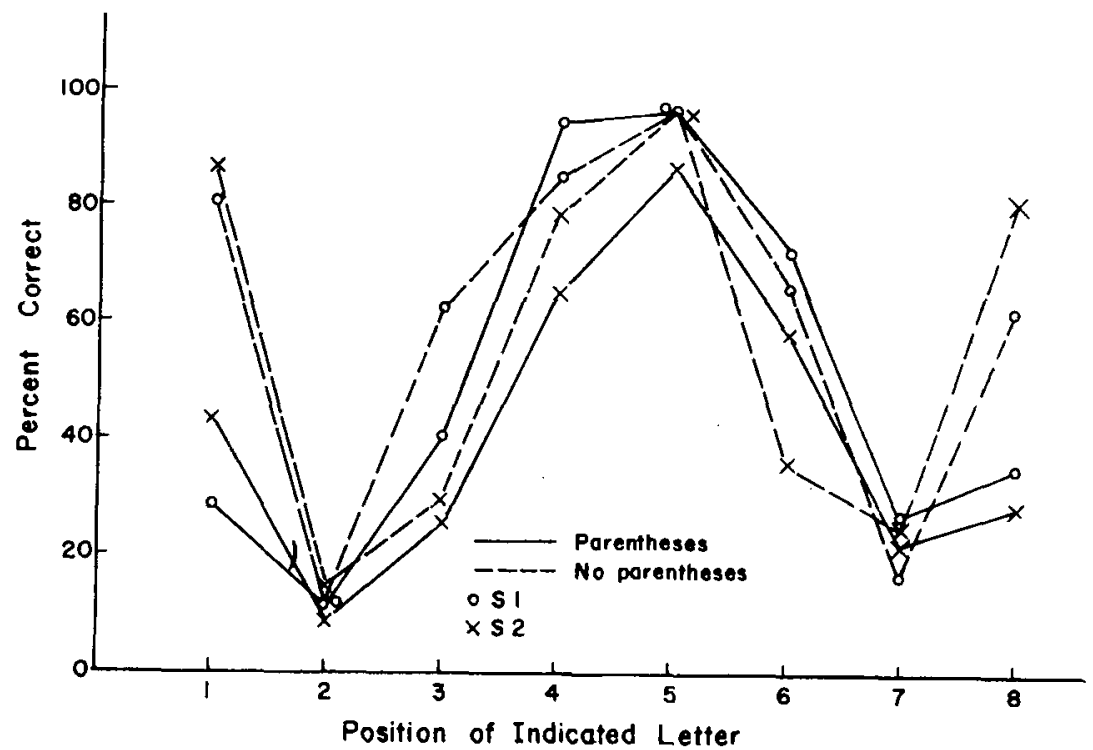

experiment may have accounted in part for of metacontrast at the ends of the array the high accuracy at the ends of the array. (elevating accuracy for those items).

Figure 3 shows that the effect of However, either the parentheses were not delaying the indicator, while highly entirely equivalent in content to the letters significant $(F=11.4, \mathrm{df}=7 / 49, \mathrm{p}<.01)$, is or metacontrast is only part of the reason not nearly as striking as that found in the for the end letter superiority, since original study. The ISI did not interact Positions 1 and 8 did not drop to very low significantly with any of the other levels of performance.

variables. This further suggests that the For encoding processes to be critical, an cause of errors rests with the initial representation (acuity) for the middle items, counteracted by metacontrast for all items except the end ones.

To determine whether or not the characteristics of the errors changed over positions, ISI, and parentheses, all letter errors were classified as visual confusions (e.g., A with N), auditory confusions (e.g., B with V), both (e.g., D with B), or neither (e.g., $X$ with 0$)^{3}$ The classifications were based upon categories from Conrad (1964), Hodge (1962), and Fisher, Monte, \& Glucksberg (1969). No significant changes in the proportion of either visual or auditory confusion errors were found over either positions or ISI, either with or without parentheses. Thus, the type of error did not appear to change as a function of the manipulations in this experiment.

\section{DISCUSSION}

Several analyses each indicate that the pattern of errors is primarily due to sensory variables and not to those that might come from encoding or memory processes. The two sensory components would seem to be acuity (elevating accuracy near the fixation point) and lack

Fig. 3. Accuracy of reporting the indicated item as a function of ISI between the array and the indicator, with and without parentheses, for two Ss. Data are summed over the eight positions. interaction between the ISI and the position of error should have been noted-positions encoded earlier being more accurate than those encoded later-thus causing the middle and right-hand positions to be relatively poorer at long delays. No such trend was found. Further, errors made in translating from a good visual storage (brief ISIs), while fewer in number, should have been visual confusions, since memory would not be overloaded, while errors made from a poor visual storage (long ISIs) should have shown a relative increase in auditory
Fig. 2. Accuracy of reporting the indicated item as a function of position, for arrays with and without parentheses, for two Ss. Data are summed over the eight ISls.

confusions. Neither of these trends was noted.

The smaller short-term visual-storage effect is puzzling, since the replication was close to the original. The two studies did differ in that the replication used only one row of letters instead of two and $20 \mathrm{ft}-\mathrm{L}$ of exposure instead of 70 . The latter change is negligible, but the former meant that $S$ had only eight letters to remember. While 8 letters exceeds the memory span for visual presentation, it does so by much less than 16 does, so that smaller decrement over time may reflect the easier task $S$ has in remembering the items.

REFERENCES

AVERBACH, E., \& CORIELL, A. S. Short-term memory in vision. Bell Telephone Technical Journal, 1961, 40, 19-31.

CONRAD, R. Acoustic confusion and immediate memory. British Journal of Psychology, 1964, $55,75-84$

FISHER, D. F., MONTE, R. A., \& GLUCKSBERG, S. Visual confusion matrices: Fact or artifact. Journal of Psychology, 1969, 31, 111-125.

HABER, R. N. An on-line computer in a visual perception laboratory. Behavioral Research Methods \& Instrumentation, 1968, 1, 86-93. HODGE, D, C. Legibility of a uniform strokewidth alphabet: I. Relative legibility of upper and lower case letters. Journal of Engineering Psychology, 1962, 1, 34-46. NOTES

1. This research was partially supported by grants from the United States Public Health Service (MH 10753) and from the National Science Foundation (GB 2909, GB 5910, and GB 4547) to the first author. We would like to thank Barbara Herr and Linda Sue Nathanson for collection of most of the data.

2. Now at Bishop's University, Lennoxville, Quebec, Canada.

3. A 25 by 25 confusion matrix of these data as well as those of several other experiments, can be obtained from the first author.

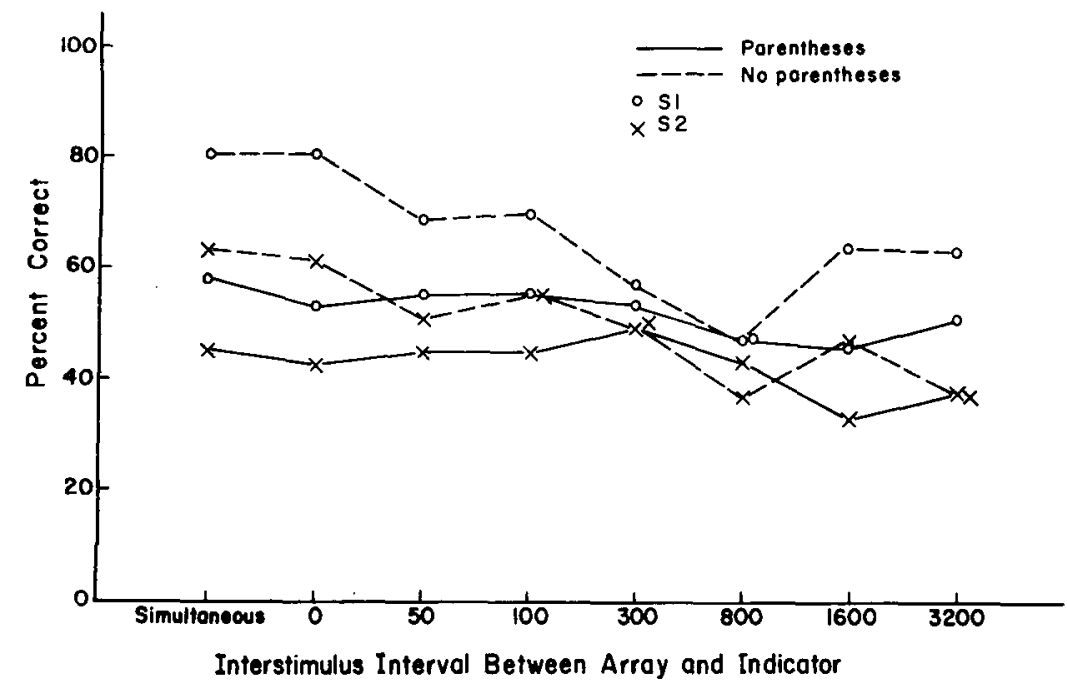

